\title{
La question des types de discours
}

Matilde Gonçalves et Audria Leal

\section{(2) OpenEdition \\ Journals}

Édition électronique

URL : http://journals.openedition.org/aes/472

DOI : $10.4000 /$ aes.472

ISSN : 2258-093X

Éditeur

Laboratoire LISAA

Référence électronique

Matilde Gonçalves et Audria Leal, «La question des types de discours », Arts et Savoirs [En ligne],

2 | 2012, mis en ligne le 15 juillet 2012, consulté le 23 avril 2019. URL : http://journals.openedition.org/ aes/472 ; DOI : 10.4000/aes.472

Ce document a été généré automatiquement le 23 avril 2019.

Centre de recherche LISAA (Littératures SAvoirs et Arts) 


\title{
La question des types de discours
}

\author{
Matilde Gonçalves et Audria Leal
}

1 Le présent travail s'intègre dans le projet de recherche Pretexto (Praxis, Conhecimento e Texto) développé par le Centre de Linguistique de l'Université Nouvelle de Lisbonne. La problématique générale de ce projet s'intéresse aux relations entre la praxis ou les pratiques humaines, les textes et l'organisation de la connaissance et se situe dans la perspective de la Théorie du Texte. Comme cadre théorique et méthodologique, nous privilégions l'Interactionnisme Socio-Discursif (ISD) ${ }^{1}$ tout en maintenant un dialogue avec d'autres courants quand cela s'avère nécessaire ${ }^{2}$.

2 L'objectif de cet article est de présenter et discuter la notion de "types de discours" développé par Bronckart. Il s'agit de»formes d'organisation linguistique, en nombre limité, dont sont composés, selon des modalités diverses, tous les genres textuels. $»^{3}$. Les types de discours correspondent à la description, d'une part, des mondes ou des plans énonciatifs et des opérations psychologiques qui y sont à la base et, d'autre part, des configurations d'unités linguistiques qui traduisent ces mondes. Ces formes linguistiques traduisent des opérations psychologiques - se reflétant dans la création de mondes discursifs spécifiques - et naissent de la relation entre les coordonnées générales organisant le contenu thématique mobilisé dans le texte et les coordonnés du monde ordinaire, c'est-à-dire de la situation d'énonciation. Deux types de relation existent : la première - de conjonction ou de disjonction - entre les coordonnées spatio-temporelles de l'action représentée et celles de l'action de langage ; et la deuxième - d'implication ou d'autonomie - entre les agents producteurs et les paramètres matériaux de l'action de langage. De là résultent quatre types de discours - interactif, théorique, la narration et le récit interactif.

Ce sont les travaux de Benveniste, notamment son étude sur les relations de temps (1959 et 1966), ceux de Weinrich (1973) et ceux de Simonin-Grumbach (1975) qui sont à la base des types de discours. Ainsi, il nous parait pertinent de cerner et de discuter cette notion, d'une part, pour rendre compte des variations qui ont suivi la pensée de Benveniste et, d'autre part, pour rendre compte des fonctions et des avantages des types de discours pour l'étude des textes et des genres textuels. 
4 Afin de mener à bien notre objectif, nous proposerons dans un premier temps de situer le cadre théorique et méthodologique dans lequel nous travaillons, puis, dans un deuxième, nous discuterons la notion de monde discursif et celle de type de discours. Finalement, nous analyserons les types de discours convoqués dans un texte empirique, faisant partie d'un corpus constitué par le groupe Pretexto.

\section{Positionnement épistémologique et méthodologique de l'ISD}

L'interactionnisme socio-discursif ${ }^{4}$ (ISD)surgit à la suite de l'interactionnisme social de Vygotsky et Mead, dans lequel sont assumés trois principes généraux : 1) traitement parallèle de la construction de la pensée consciente et de la problématique de la construction des faits sociaux et culturels en tant que deux versants du développement humain ; 2) questionnement des Sciences Humaines sur le corpus de la philosophie de l'esprit (d'Aristote à Marx) et prise en considération des problèmes d'intervention pratique (notamment dans le champ scolaire) ; 3) contestation de la division des sciences humaines en de multiples disciplines et sous-disciplines (conséquence du positivisme de Comte).

6 Héritier de l'interactionnisme social, et cherchant donner suite à celui-ci, l'ISD se propose de contribuer pour une science de l'humain. Pour cela, ce vaste courant convoque la linguistique, la psychologie, la sociologie et la philosophie, de manière à appréhender la complexité des phénomènes langagiers humains.

7 La particularité de l'ISD réside dans le fait d'assumer que le langage joue un rôle essentiel tant au niveau du développement et du fonctionnement psychique qu'au niveau du développement des activités collectives. Notons que ces activités constituent l'espace d'organisation et de médiation entre les êtres humains et l'environnement (Leontiev 1979).

En accord avec la thèse partagée par Saussure et Vygotsky, selon laquelle les signes langagiers fondent la constitution de la pensée consciente, l'ISD vise démontrer que les pratiques de langage situées (textes et discours) sont les principaux instruments pour le développement humain, au niveau des connaissances et des savoirs et au niveau des capacités de l'agir et de l'identité des personnes.

Partant du rôle prépondérant des pratiques langagières dans le développement humain, l'approche de l'ISD est descendante, en d'autres termes, elle part des pratiques sociales vers les activités de langage, de celles-ci vers les genres de texte, des genres vers les textes, et des textes vers les unités linguistiques. De plus, l'ISD se base sur deux notions, l'action langagière et le modèle de l'architecture textuelle, présenté dans plusieurs travaux ${ }^{5}$ : «Les produits base de l'ISD sont d'une part le modèle de l'action langagière qui vise à conceptualiser les conditions synchroniques des conditions de production des textes et, le modèle de l'architecture textuelle. $\rrbracket^{6}$ Quelques mots sur ces deux notions s'imposent. Les textes s'insèrent toujours dans une activité de langage ${ }^{7}$ et ont une incidence dans le développement et dans l'agir humain. Ils matérialisent l'action de langage qui est une unité psychologique abstraite correspondant aux représentations que l'agent a du contexte d'action. L'activité de langage se manifeste et agit à un niveau collectif, alors que l'action de langage se situe à un niveau individuel. 

est décrit comme un feuilleté constitué de trois niveaux organisationnels - les mécanismes de prise en charge énonciative, les mécanismes de textualisation et l'infrastructure générale. Ces trois niveaux se superposent les uns aux autres et interagissent entre eux. Les mécanismes énonciatifs correspondent au niveau extérieur. Viennent ensuite les mécanismes de textualisation et enfin l'infrastructure générale qui correspond au niveau le plus profond de l'architecture textuelle.

11 Présentons de manière succincte, les éléments appartenant aux trois niveaux organisationnels ${ }^{8}$. Les mécanismes énonciatifs dépendent de la situation de communication, d'où son appartenance au niveau superficiel, et englobent la « responsabilité énonciative », la « distribution des voix » et les « attributions modales». Les mécanismes de textualisation sont en rapport avec la progression du contenu thématique du texte et englobent la connexion, la cohésion nominale. Terminons par l'infrastructure générale, correspondant au niveau le plus profond de l'architecture et réunissant le contenu thématique et les types de discours.

Les types de discours sont observables dans les différents segments qu'un texte comprend et équivalent à des formes d'organisation linguistique. Ils correspondent à la matérialisation linguistique des mondes discursifs. Dans ce qui suit, nous nous proposons de voir de manière plus approfondie les mondes discursifs et les types de discours.

\section{Construction des mondes discursifs et types de discours}

\section{Les mondes discursifs}

Dans la perspective de Bronckart, la production langagière incorpore obligatoirement deux versants : un linguistique et un autre psychologique. Dans son versant linguistique, nous avons les types de discours, qui comme nous l'avons défini précédemment, sont des segments constitutifs des textes et présents de manière organisée dans les genres textuels. Dans l'autre versant, ces formes linguistiques représentent des opérations psychologiques qui reflètent la création de mondes discursifs spécifiques. Ainsi, à un niveau cognitif, l'activité langagière repose sur des opérations d'ordre psychologique qui engendrent la création de mondes discursifs. Comme le souligne l'auteur :

[...] en raison même de sa nature sémiotique, l'activité langagière se soutient nécessairement de la création de mondes virtuels. Ces mondes sont des systèmes de coordonnées formelles, qui d'une part sont radicalement «autres» que les systèmes de coordonnés des mondes représentés dans lesquels se déploient les actions d'agents humains, mais qui d'autre part doivent exhiber le type de rapport qu'ils entretiennent avec ces mondes de l'activité humaine. Par convention, nous qualifierons les mondes représentés des agents humains de monde ordinaire et les mondes virtuels créés par l'activité langagière de mondes discursifs. ${ }^{9}$

En effet, les mondes discursifs sont sémiotisés dans les textes par les types de discours. En d'autres termes, les types de discours sont des manifestations linguistiques de ces mondes psychologiques. Bronckart part de trois études importantes sur les verbes d'une langue naturelle pour déterminer la notion de types de discours. La première étude qui aura une grande influence dans la conception théorique des types de discours est celle de Benveniste $(1959 / 1966)^{10}$ sur les relations des temps verbaux du français. Le linguiste 
affirme que les temps verbaux se divisent en deux sous-systèmes différents mais qui se complètent, formant ainsi deux plans énonciatifs distincts : le plan de l'histoire et le plan du discours. La deuxième étude appartient à Weinrich (1973) ${ }^{11}$ et établit une distinction entre les temps commentatifs et les temps narratifs et une opposition entre le monde commenté et le monde raconté. L'étude de Simonin-Grumbach $(1975)^{12}$, qui correspond à la troisième étude, cherche à identifier les unités linguistiques caractéristiques et distinctives des mondes ou plans énonciatifs afin de décrire les opérations psychologiques sous-jacentes à ces mondes; à ces unités, l'auteure donne le nom de type de discours. Nous pouvons donc affirmer que l'approche de Bronckart se situe dans la continuité de ces travaux, tout en mettant en relation les idées des trois auteurs et en amplifiant leur portée dans la concrétisation de sa propre théorie, comme nous pouvons le voir dans la citation suivante :

Notre démarche propre se situe dans la continuité de ces travaux, en ce qu'elle consiste à décrire, d'une part les mondes ou plans d'énonciation ainsi que les opérations psychologiques qui les sous-tendent, et d'autre part les configurations d'unités linguistiques "traduisant" ces mondes dans le cadre d'une langue naturelle donnée. ${ }^{13}$

Deux questions sont centrales dans la notion des mondes discursifs. La première fait référence à l'affirmation selon laquelle les mondes discursifs sont des représentations du monde réel, c'est-à-dire, le monde dans lequel se développent les actions des agents producteurs. La deuxième est une conséquence de la première. Ces mondes discursifs sont construits sur la base de deux sous-ensembles d'opérations : les premières font référence à la relation entre les coordonnées qui organisent le contenu thématique et les coordonnées du monde réel. Cette relation est dite de conjonction quand les coordonnées spatio-temporelles de l'action représentée sont tenues comme conjointes de celles de l'action de langage, ou, au contraire, la relation peut être de disjonction, quand les coordonnées spatio-temporelles se posent dans un "ailleurs». Le deuxième sousensemble d'opérations éclaire la mise en relation des différentes instances d'agentivité (personnages, groupes, institutions, etc.) et son inscription spatio-temporelle avec les paramètres physiques de l'action de langage en cours (agent producteur, interlocuteur et espace-temps de production). Ainsi, il pourra y avoir une relation d'implication ou d'autonomie entre les agents producteurs et les paramètres matériaux de l'action de langage. Ces deux sous-ensembles d'opérations se combinent en deux grands groupes: l'ordre de l'exposer et l'ordre du raconter. Ces derniers engendrent, à leur tour, quatre mondes discursifs : monde de l'exposer impliqué ; monde de l'exposer autonome ; monde du raconter impliqué ; monde du raconter autonome.

\section{Les quatre types de discours}

À partir de la construction des mondes discursifs, Bronckart (1997/2008) propose l'existence de quatre types de discours, à savoir: le discours interactif (monde de l'exposer impliqué); le discours théorique (monde de l'exposer autonome); le récit interactif (monde du raconter impliqué) et la narration (monde du raconter autonome), comme nous pouvons l'observer dans le tableau ci-dessous : 


\begin{tabular}{|c|c|c|c|}
\hline & & \multicolumn{2}{|c|}{ Organisation temporelle } \\
\hline & & Conjonction & Disjonction \\
\hline & & $\begin{array}{l}\text { Ordre de } \\
\text { I'EXPOSER }\end{array}$ & $\begin{array}{l}\text { Ordre du } \\
\text { RACONTER }\end{array}$ \\
\hline \multirow{2}{*}{$\begin{array}{l}\text { Organisation } \\
\text { actorielle }\end{array}$} & Implication & Disc ours interactif & Récit interactif \\
\hline & Autonomie & Discours théorique & Narration \\
\hline
\end{tabular}

Soulignons que le choix des types de discours par l'agent producteur est conditionné par l'interprétation que celui-ci a de la situation communicative dans laquelle le texte est créé. En accord avec le modèle, la production langagière se concrétise dans un texte empirique, combinant normalement plusieurs types de discours. Les types de discours s'étendent successivement soit dans les oraux ou les textes écrits et sont sous-jacents à une activité qui est délimitée par l'action de langage. Cette action se déploie dans une certaine durée, formelle ou psychologie, qui est qualifiée d'axe de référence temporelle d'un type de discours ${ }^{15}$. De plus, l'activité langagière est caractérisée par les instances agentives du texte qui peuvent manifester un rapport d'identité ou de différence avec les instances de l'action de langage ${ }^{16}$ ou impliquer des éléments de l'action langagière qui se traduisent dans l'emploi des formes pronominales avec valeur déictique.

Le discours interactif se caractérise par la " présence d'unités renvoyant à l'interaction verbale elle-même, qu'elle soit réelle ou mise-en-scène, et au caractère conjoint-impliqué du monde discursif créé $\aleph^{17}$. Les marques linguistiques de l'organisation spatio-temporelle qui indiquent la conjonction de ce type de discours sont les temps verbaux du présent de l'indicatif, du passé composé et du futur périphrastique et la présence de déictiques temporels tels que "maintenant, à présent, tout à l'heure». Quant à son organisation actorielle, celle-ci est marquée par la présence de noms propres, de pronoms et d'adjectifs de première, deuxième personne singulier/pluriel (je, tu, nous, vous).

On identifie le discours théorique par la présence d'unités linguistiques qui présentent des caractéristiques conjointes-autonomes du monde construit. Dans l'organisation spatio-temporelle, nous retrouvons comme temps verbaux le présent de l'indicatif, le passé composé, le conditionnel et une absence quasi-totale des formes du futur. L'organisation actorielle est marquée par l'absence d'unités renvoyant directement aux interactants ou à l'espace-temps de la production, par l'absence de noms propres et aussi par l'absence de pronoms et d'adjectifs de première, seconde personne singulier/pluriel et par la possible présence du pronom on.

Le récit interactif est déterminé par la présence d'unités linguistiques qui présentent des caractéristiques disjointes-impliquées du monde construit. L'organisation spatiotemporelle est marquée par deux temps verbaux dominants: le passé composé et l'imparfait, mais aussi par le passé simple, plus-que-parfait, futur et conditionnel. Nous retrouvons aussi dans ce type de discours la présence d'autres types d'organisateurs temporels qui marquent l'origine spatio-temporelle, comme "l'autre jour», «quand 
j'avais quinze ans ». L'organisation actorielle est définie par la présence de pronoms et d'adjectifs de première, seconde personne singulier/pluriel qui renvoient directement aux protagonistes de l'interaction verbale.

21 Finalement, la narration est déterminée par la présence d'unités linguistiques qui manifestent des caractéristiques disjointes-autonomes du monde textuellement construit. Les marques de l'organisation spatio-temporelle sont les temps du soussystème des temps de l'histoire et les temps narratifs - le passé simple, l'imparfait, le passé antérieur, le plus-que-parfait et le conditionnel - mais aussi d'autres organisateurs temporels qui marquent l'origine spatio-temporelle explicitée. L'organisation actorielle est caractérisée par l'absence d'unités renvoyant directement à l'agent producteur et à ses destinataires.

Comme nous pouvons l'observer, les deux opérations qui constituent les types de discours semblent reposer sur des décisions de caractère binaire : soit au niveau de la conjonction/ disjonction, soit au niveau de l'implication/autonomie. Ces deux opérations sont facilement observables en types discursifs " purs ». Nonobstant, on pourra retrouver des segments de texte dans lesquels se manifestent des superpositions, des imbrications, voire même des fusions de types de discours - comme nous le verrons dans l'exemple textuel analysé postérieurement, dans lequel, dans l'ordre de l'exposer, on retrouvera un type de discours mixte, le discours interactif - théorique, qui comporte des caractéristiques aussi bien du discours interactif que du discours théorique. Dans ce cas, le principe binaire n'est pas respecté. Bronckart ${ }^{18}$ assume que les types de discours présentent des variantes, des frontières et voire même des fusions entre eux dues à la variation de la situation de production. C'est pour cela que, dans le tableau, les lignes qui séparent les types de discours sont en pointillés. Toutefois, ceci ne remet pas en cause la définition des types de discours ni les descriptions de ses unités constitutives.

\section{Types de discours dans une perspective praxiologique : un exemple de l'ordre de l'exposer}

23 Nous travaillons sur des textes empiriques qui circulent en société - élément central du positionnement de l'ISD - et pour ce travail, nous avons choisi un extrait du site web d'un producteur de vin portugais Aveleda ${ }^{19}$. Ce texte fait partie du corpus développé au sein de notre projet de recherche Pretexto (Praxis, Texte et Connaissance). Le choix d'un site web s'attache à deux facteurs. Tout d'abord, les textes numériques jouent de plus en plus un rôle prépondérant au sein de la société, puis parce qu'étant des textes appartenant à des genres textuels récents et dont les caractéristiques ne sont pas totalement stabilisées, ces textes peuvent soulever des questionnements quant aux instruments linguistiques développés pour décrire et rendre compte des textes historiquement établis. Par ailleurs, le texte date du 26 octobre 2008, il faut donc ancrer son contenu à cette date. De fait, le contenu thématique disponible sur le site, à la date de décembre 2011 diverge quelque peu de celui que nous présentons ici. 


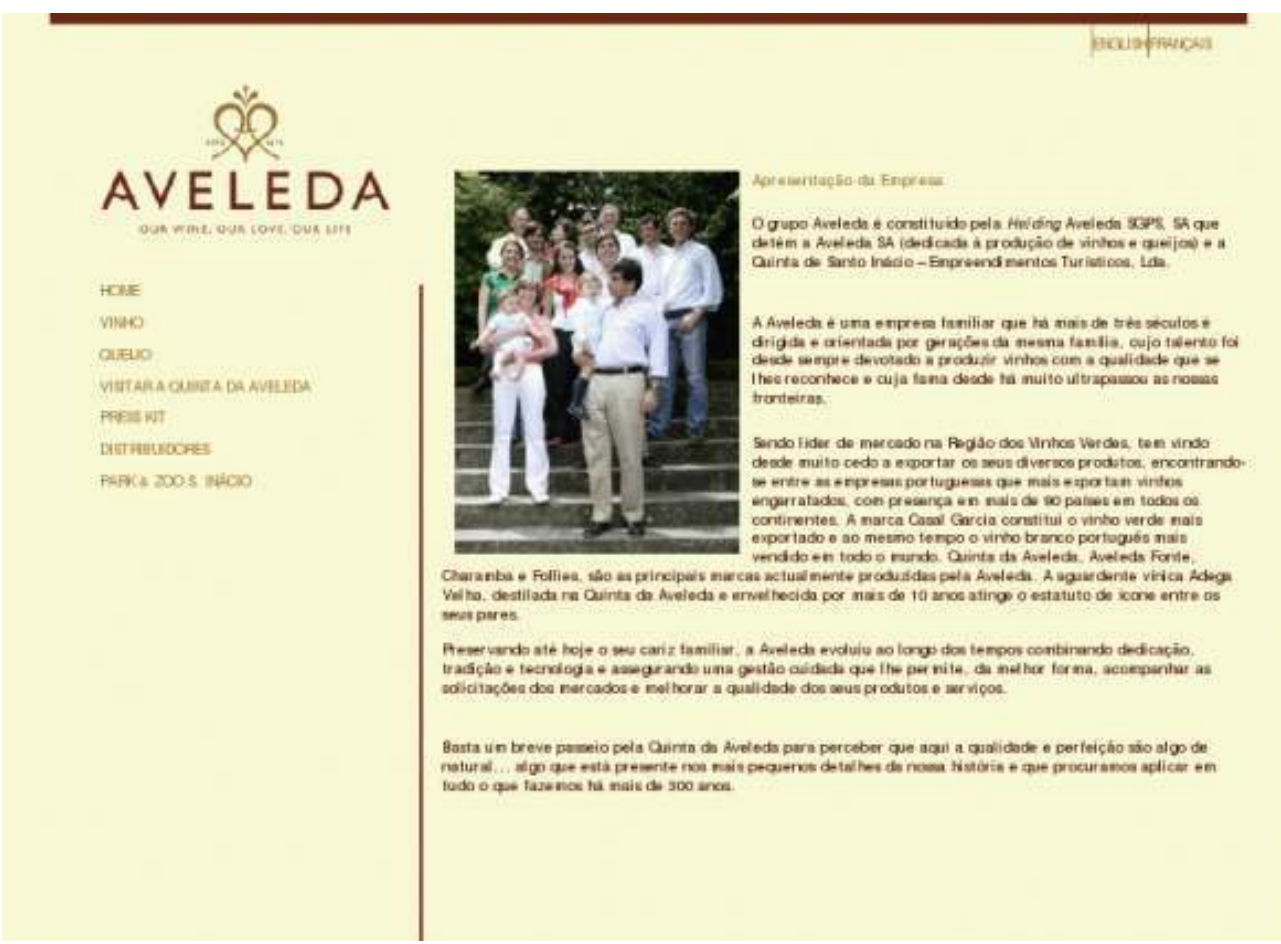

http://www.aveledaportugal.pt/ (consulté le 26 octobre 2008)

«O grupo Aveleda é constituído pela Holding Aveleda SGPS. SA que detém a Aveleda SA (dedicada à produção de vinhos e queijos) e a Quinta de Santo Inácio - Empreendimentos Turísticos, Lda.

Le groupe Aveleda constitué par la Holding Aveleda SGPS.SA détient Aveleda SA (dédiée à la production de vins et de fromages) et la Quinta de Santo Inácio ${ }^{20}$ - Entreprises touristiques, SARL ».

Dans le premier paragraphe, nous pouvons observer la prédominance du monde discursif de l'ordre de l'exposer, et, en particulier, le discours théorique ${ }^{21}$. En effet, la présence du présent de l'indicatif du verbe «é » (est) et du verbe «detém » (détient), à la troisième personne du singulier - pointe une conjonction temporelle entre le monde discursif et le monde ordinaire (ou la situation d'énonciation). L'absence de marques de première et de deuxième personne du singulier et du pluriel renvoie à une autonomie de l'agent producteur du texte, comme nous pouvons le voir dans ce qui suit.

\section{Paragraphe 2 :}

"A Aveleda é uma empresa familiar que há mais de três séculos é dirigida e orientada por gerações da mesma família, cujo talento foi desde sempre devotado a produzir vinhos com 
a qualidade que se lhes conhece e cuja fama há muito ultrapassou as nossas fronteiras.

Aveleda est une entreprise familiale, qui depuis plus de trois siècles est dirigée et guidée par des générations de la même famille, dont le talent s'est dévoué depuis toujours à produire des vins de qualité reconnue et dont la renommée a depuis longtemps dépassé nos frontières ».

Dans le deuxième paragraphe, nous sommes toujours dans l'ordre de l'exposer, puisqu'il n'y a pas de disjonction avec la situation d'énonciation, comme le manifeste le présent de l'indicatif «é » (est) du verbe "ser » (être). Les marques temporelles telles que « há mais de três séculos » (depuis plus de trois siècles), « desde sempre », (depuis toujours), « há muito» (depuis longtemps) marquent aussi une conjonction avec la situation d'énonciation, et ce, parce que même si ces unités possèdent une origine temporelle dans le passé l'action se prolonge dans le présent. Intégré dans ces segments de discours théorique, nous retrouvons une marque du discours interactif : la première personne du pluriel avec la présence du pronom personnel «nossas» (notres). Celle-ci implique la présence de l'agent producteur et du lecteur/récepteur.

\section{Paragraphe 3 :}

«Sendo líder de mercado na Região dos Vinhos verdes, tem vindo desde muito cedo a exportar os seus diversos produtos, encontrando-se entre as empresas portuguesas que mais exportam vinhos engarrafados, com presença em mais de 90 países em todos os continentes. A marca Casal Garcia constitui o vinho verde mais exportado e ao mesmo tempo o vinho branco português mais vendido em todo o mundo. Quinta da Aveleda, Aveleda Fonte, Charamba e Follies, são as principais marcas actualmente produzidas pela Aveleda. A aguardente vínica Adega Velha, destilada na Quinta da Aveleda e envelhecida por mais de 10 anos atinge o estatuto de ícone entre os seus pares ».

Étant le leader du marché dans la Région des Vins Verts, [Aveleda] a commencé très tôt à exporter ses divers produits, se trouvant parmi les entreprises qui exportent le plus de vins mis en bouteille, marquant présence dans plus de 90 pays dans les cinq continents. La marque Casal Garcia constitue le vin vert le plus exporté et en même temps le vin blanc portugais le plus vendu au monde. Quinta da Aveleda, Aveleda Fonte, Charamba et Follies sont les principales marques actuellement produites par Aveleda. L'eau de vie vinicole Adega Velha, distillé à Quinta da Aveleda et vieillie pendant plus de dix ans, atteint son statut d'icône entre ses pairs.

Le paragraphe 3 suit le même principe temporel que nous avons vu dans le paragraphe précédent. En effet, les marqueurs temporels «tem vindo desde muito cedo» (a commencé très tôt), «por mais de dez anos » (pour plus de dix ans) ont leur origine dans le passé et se prolongent dans le présent. Encore dans ce paragraphe, les verbes « exportam» (exportent), «constitui» (constitue) «são» (sont) renforcent la construction d'un monde discursif conjoint. Comme dans le premier paragraphe, il n'y a pas de marques d'agentivité impliquée, nous nous donc face à du discours théorique.

\section{Paragraphe 4 :}

«Preservando até hoje o seu cariz familiar, a Aveleda evoluiu ao longo dos tempos combinando dedicação, tradição e tecnologia e assegurando uma gestão cuidada que lhe permite, da melhor forma, acompanhar as solicitações dos mercados e melhorar a qualidade dos seus produtos e serviços.

Préservant jusqu'à nos jours son caractère familial, Aveleda a évolué au long des temps alliant dévouement, tradition et technologie, tout en assurant une gestion soignée qui lui 
permet d'accompagner les demandes du marché et d'améliorer la qualité de ses produits et de ses services de la meilleure manière ».

Dans le quatrième paragraphe, signalons deux points intéressants. Le premier est la présence du déictique « hoje » (aujourd'hui). Le déictique est un marqueur par excellence $\mathrm{du}$ discours interactif puisqu'il indique une relation directe avec la situation réelle d'énonciation. Dans ce cas précis, «hoje " (aujourd'hui) associé au verbe au gérondif «preservando» (préservant) dans l'expression "preservando até hoje» montre une valeur temporelle de continuité (du passé vers le présent). Cette même valeur est renforcée par l'expression " ao longo dos tempos" (au long des temps) qui confère une conjonction temporelle malgré la présence du verbe au passé simple (pretérito perfeito) « evoluiu » (évolua) et qui, normalement, indique une valeur de disjonction.

\section{Paragraphe 5 :}

«Basta um breve passeio pela Quinta da Aveleda para perceber que aqui a qualidade e perfeição são algo de natural... algo que está presente nos mais pequenos detalhes da nossa história e que procuramos aplicar em tudo o que fazemos há mais de 300 anos.

Il suffit d'une brève promenade dans la "Quinta » Aveleda pour comprendre que la qualité et la perfection sont, ici, quelque chose de naturel... quelque chose qui est présent dans les plus petits détails de notre histoire et que nous cherchons à appliquer dans tout ce que nous faisons depuis près de 300 ans ».

Dans le dernier paragraphe, nous attirons l'attention sur les marques d'agentivité des acteurs de l'interaction caractérisés par la présence du pronom possessif "nossa » (notre) et sur les formes verbales à la première personne du pluriel "procuramos " (cherchons) et «fazemos » (faisons). Nous remarquons aussi la présence d'un déictique spatial «aqui » (ici), qui, comme dans le paragraphe précédent, renforce la marque d'implication qui manifeste la présence du discours interactif, dans une séquence de discours théorique. Ainsi, nous pouvons dire que le deuxième et le dernier paragraphe révèlent une fusion entre le discours théorique et le discours interactif, formant ainsi le discours mixte interactif- théorique (Bronckart, 1997 : 189-211).

31 Comme nous l'avons vu dans le texte analysé, on retrouve le discours théorique et le discours mixte théorique interactif. Le recours à ces types de discours s'explique par la dimension praxéologique du texte. En effet, si l'on pense aux objectifs du texte, on comprend qu'à travers le discours théorique l'agent producteur veut donner une image claire et objective de l'entreprise Aveleda, alors que les marques du discours interactif suggèrent une proximité avec le lecteur de manière à l'impliquer et à le convaincre du sérieux, de l'histoire et de l'expérience du groupe Aveleda.

\section{Conclusion}

Afin de répondre au sujet lancé par le colloque «Les théories énonciatives aujourd'hui : Benveniste après un demi-siècle », nous nous sommes proposés de présenter la notion de type de discours développé par Bronckart au sein de l'interactionnisme socio-discursif. Notre objectif cherchait à mettre en avant une des variations qui a découlé de la pensée et des travaux de Benveniste.

Ainsi, dans un premier temps, nous nous sommes attachés à poser le cadre théorique et méthodologique de l'ISD et au sein duquel nous travaillons, puis nous avons focalisé les différents mondes discursifs et leur relation avec les types de discours et avons donc posé 
le fait que les types de discours sont la matérialisation linguistique des mondes discursifs. Finalement, l'analyse des types de discours présents dans un texte empirique, nous a permis de voir que les frontières entre les types du discours ne sont pas hermétiques. En effet, il peut y avoir des types mixtes comme nous l'avons vu lors de l'analyse. De plus, l'observation des types de discours en situation réelle a mis en avant le fait qu'il n'existe pas de type de discours théorique " pur » car l'existence de noms propres indique un lien avec les instances agentives de l'action de langage.

Ces deux points confortent d'une part la nécessité de travailler avec des textes réels, concrets car ils mettent au défi les instruments théoriques et permettent ainsi d'avancer dans la construction d'outils performants qui permettent de décrire et comprendre la réalité linguistique et d'autre part, qu'il faut savoir ou apprendre à faire face à la complexité telle que Edgar Morin l'a décrite, c'est-à-dire, gérer le connu et l'inconnu, ce que l'on peut expliquer et ce que l'on ne peut expliquer, sans toutefois minimiser les catégorisations ou les typologies. De fait, elles peuvent être une manière de décrire et comprendre les objets complexes.

De plus, selon notre perspective, pour une compréhension authentique du phénomène langagier, il importe de comprendre l'interaction humaine qui se réalise à travers les textes complexes et empiriques qui circulent au quotidien.

\section{BIBLIOGRAPHIE}

ADAM Jean-Michel, Linguistique textuelle. Des genres de discours aux textes, Paris, Nathan, 1999.

BRONCKART Jean-Paul, Activité langagière, textes et discours. Pour un interactionnisme socio-discursif, Lausanne, Éditions Delachaux et Niestlé, 1997.

BRONCKART Jean-Paul, « Les différentes facettes de l'interactionnisme socio-discursif » in Calidoscópio, vol. 3, nº3, UNISINOS (Brésil), 2005, p. 143-159.

BRONCKART Jean-Paul, Atividade de Linguagem, Discurso e Desenvolvimento Humano, Anna Rachel Machado et Maria de Lourdes Meirelles (dir.), Campinas, Mercado de Letras, 2006.

BRONCKART Jean-Paul, « Genre de textes, types de discours et "degrés” de langue », in Texto!

Janvier, vol. XIII, 2008. Voir l'article en ligne sur internet : http://www.revue-texto.net/ index.php?id=86.

GONÇALVES Mathilde, La fragmentation dans la littérature portugaise contemporaine : indices énonciatifs, configurations textuelles et parcours interprétatifs, Lille, A.N.R.T, Thèses à la carte, 2010.

LEAL Audria \& GONÇALVES, Matilde, «A Interação em Gêneros Textuais : Do Cartoon ao Web-site » in Anais do II ${ }^{\circ}$ Congresso Internacional de Linguagem e Interação, São Leopoldo. Brasil, 2010.

LEAL Audria, «O papel do discurso teórico nos cartoons » in Estudos Linguísticos/Linguistic Studies, Lisbonne, Edições Colibri, nº 5, juillet 2010, p. 223-245.

LEAL Audria, «A presença do discurso interativo no género textual cartoon » in Estudos Linguísticos/Linguistic Studies, Lisbonne, Edições Colibri, no 1, juillet 2008, p. 71-80. 
MAINGUENEAU Dominique, Analyser les textes de communication, Paris, Dunod, 1998.

RASTIER François, Arts et sciences du texte, Paris, PUF, 2001.

\section{NOTES}

1. Voir à ce propos, Jean-Paul Bronckart, Activité langagière, textes et discours. Pour un interactionnisme socio-discursif, Lausanne, Éditions Delachaux et Niestlé, 1997 et Atividade de Linguagem, Discurso e Desenvolvimento Humano, Anna Rachel Machado et Maria de Lourdes Meirelles (dir.), Campinas, Mercado de Letras, 2006. Et aussi l'article « Genre de textes, types de discours et "degrés" de langue", in Texto! Janvier, vol. XIII, 2008. Cf. l'article en ligne sur internet : http://www.revue-texto.net/index.php?id=86.

2. Jean-Michel Adam, Linguistique textuelle. Des genres de discours aux textes, Paris, Nathan, 1999 ; Dominique Maingueneau, Analyser les textes de communication, Paris, Dunod, 1998 ; François Rastier, Arts et sciences du texte, Paris, PUF, 2001.

3. Jean-Paul Bronckart, Activité langagière, textes et discours. Pour un interactionnisme socio-discursif, op. cit., p. 254.

4. Jean-Paul Bronckart, Activité langagière, textes et discours. Pour un interactionnisme discursif, op. cit.

5. Ibid. Voir également à ce propos, Jean-Paul Bronckart, «Genre de textes, types de discours et “degrés" de langue ", op. cit.

6. Bronckart, Jean-Paul, "Les différentes facettes de l'interactionnisme socio-discursif» in Calidoscópio, vol. 3, no3, UNISINOS (Brésil), 2005, p. 143-159.

7. L'activité de langage est définie par J-P. Bronckart et $\mathrm{K}$. Stroumza comme : "phénomène collectif d'élaboration et de mise en circulation de textes, dont la visée ultime est d'établir une entente sur ce que sont les contextes et les propriétés des activités en général » («Les types de discours comme traces cristallisées de l'action du langage » in Roulet, E. \& Burger, M. Les modèles $d u$ discours au défi d'un "dialogue romanesque " : l'incipit de roman de R. Pinget, Éditions Le Libera, Nancy, 2002, p. 223.

8. Pour plus de détails nous renvoyons à Bronckart, op. cit.

9. Jean-Paul Bronckart, Activité langagière, textes et discours. Pour un interactionnisme socio-discursif, op. cit., p. 153.

10. Émile Benveniste, «Les relations de temps dans le verbe français » [1956], Bulletin de la Société de linguistique, n. 54. Réédité en Problèmes de linguistique générale, t. I, Paris, Gallimard, 1966, p 237-250.

11. Voir à ce propos, Harald Weinrich, Le temps, Paris, Éditions du Seuil, 1973.

12. Jenny Simonin-Grumbach, "Pour une typologie des discours » in Julia Kristeva, Jean-Claude Milner et Nicolas Ruwet (dir.), Langue, discours, société. Pour Émile Benveniste, Paris, Éditions du Seuil, p. 85-121.

13. Jean-Paul Bronckart, Activité langagière, textes et discours. Pour un interactionnisme discursif, op. cit., p. 152-153.

14. Jean-Paul Bronckart, "Genre de textes, types de discours et "degrés" de langue », op. cit., p. 71.

15. Ibid., p. 64.

16. Ibid., p. 69.

17. Jean-Paul Bronckart, Activité langagière, textes et discours. Pour un interactionnisme discursif, op. cit., p 170.

18. Ibid., p. 189.

19. http://www.aveleda.pt (consulté le 26 octobre 2008). 
20. Une "Quinta" au Portugal a plusieurs sens. Dans ce contexte, "Quinta " correspond à une exploitation vinicole et trouve un équivalent en français dans « Château » ou " Domaine ».

21. Notons, toutefois, que la présence des noms propres «Aveleda », « Holding Aveleda SGPS », «Quinta de Santo Inácio» attestent une trace d'implication avec la situation, comme nous le verrons postérieurement.

INDEX

Mots-clés : Bronckart (Jean-Paul), discours, organisation linguistique

\section{AUTEURS}

\section{MATILDE GONÇALVES}

FCT, CLUNL, Lisbonne

AUDRIA LEAL

CLUNL, Lisbonne 